Annuaire du Collège de France 2017-2018

\title{
Françoise Héritier (1933-2018)
}

\section{Philippe Descola}

\section{OpenEdition}

\section{Journals}

Édition électronique

URL : https://journals.openedition.org/annuaire-cdf/16629

DOI : 10.4000/annuaire-cdf.16629

ISBN : 978-2-7226-0572-5

ISSN : 2109-9227

Éditeur

Collège de France

Édition imprimée

Date de publication : 30 décembre 2020

Pagination : 737-739

ISBN : 978-2-7226-0516-9

ISSN : 0069-5580

Référence électronique

Philippe Descola, «Françoise Héritier (1933-2018) », L'annuaire du Collège de France [En ligne], 118| 2020, mis en ligne le 01 avril 2021, consulté le 22 août 2022. URL : http://journals.openedition.org/ annuaire-cdf/16629; DOI : https://doi.org/10.4000/annuaire-cdf.16629 


\section{FRANÇOISE HÉRITIER (1933-2018)}

Née le 15 novembre 1933 à Veauche, dans la Loire, Françoise Héritier s'est éteinte à Paris le 15 novembre 2017, le jour de ses 84 ans. D'une enfance passée en Auvergne et de la fréquentation régulière du monde paysan d'où étaient issus ses grands-parents, elle a gardé toute sa vie un goût pour le langage de la terre et des saisons, une familiarité avec les techniques manuelles, en même temps qu'un sens aigu des différences de comportement et de statut éveillé par son observation d'une société rurale où prévalaient encore le droit d'aînesse, la solidarité familiale dans des parentèles étendues et une nette séparation entre les univers masculins et féminins.

Montée à Paris dans l'adolescence suite à un changement de situation de ses parents, Françoise Héritier y entreprend des études d'histoire et de géographie à la Sorbonne, et c'est par hasard qu'elle découvre l'ethnologie en suivant le cours de Claude Lévi-Strauss à la section des sciences religieuses de l'École pratique des hautes études (EPHE) - un enseignement dont elle dira plus tard qu'il fut la révélation de sa vie. Il imprima en tout cas à celle-ci un cours nouveau puisque, après avoir passé un certificat d'ethnologie, Françoise Héritier est engagée sur la recommandation de Lévi-Strauss par le gouvernement général de l'Afrique-Occidentale française afin de mener, en compagnie de son condisciple et futur époux Michel Izard, une enquête démographique et géographique préparatoire à la construction d'un barrage sur un affluent de la Volta, une expérience d'un an qui va décider de sa carrière d'africaniste. C'est au cours de cette étude, en effet, que la jeune étudiante découvre à la fois sa vocation d'ethnologue et le pays samo, qui deviendra son terrain de prédilection. Françoise Héritier accomplit alors en Afrique-Occidentale à partir de 1963 une série de séjours totalisant près de cinq années de travail de terrain, principalement auprès des Samo, mais aussi des Pana et des Mossi, dans ce qui deviendra le Burkina Faso et, de façon plus ponctuelle, chez les Dogon du Mali.

Au retour de sa première mission, Françoise Héritier est nommée chef de travaux à la VIe section de l'EPHE, où elle dirige de 1961 à 1966 le Centre d'analyse documentaire pour l'Afrique noire. Elle entre en 1967 au Centre national de la recherche scientifique (CNRS) et vient travailler auprès de Claude Lévi-Strauss au Laboratoire d'anthropologie sociale du Collège de France que celui-ci avait fondé en 1960. Élue en 1980 directeur d'études à l'École des hautes études en sciences sociales (EHESS), elle continue d'exercer cette fonction lorsqu'elle est élue professeur au Collège de France dans la chaire Étude comparée des sociétés africaines qu'elle occupe de 1982 à 1999 en même temps qu'elle dirige le Laboratoire d'anthropologie sociale. 
Les recherches et l'enseignement de Françoise Héritier se sont orientés dans deux directions principales et complémentaires : l'analyse des systèmes de parenté et du fonctionnement des structures d'alliance de mariage, d'une part, et l'étude de la symbolique du corps dans la définition des identités individuelles et collectives, d'autre part. Dans l'un et l'autre cas, c'est au premier chef la volonté de rendre raison des institutions qu'elle décrivait dans ses enquêtes en Afrique qui l'a conduite à développer des théories dont la portée est beaucoup plus générale. Chez les Samo de Haute-Volta, en effet, Françoise Héritier découvrait un système de parenté du type que Claude Lévi-Strauss avait baptisé de «semi-complexe», mais dont il avait différé l'analyse. Par contraste avec les systèmes de parenté dits "élémentaires », auxquels Lévi-Strauss avait consacré sa thèse de doctorat d'État, lesquels reposent sur des prescriptions de mariage avec des catégories de parents définis soit par une relation généalogique soit par leur appartenance à une classe, les systèmes de parenté dits « semi-complexes » reposent sur des interdictions de prendre un conjoint dans des unités sociales - clans ou lignages - définies par rapport à l'individu de référence ; quant aux systèmes dits « complexes »-ceux qui caractérisent la parenté dans l'Europe contemporaine, par exemple -, ils reposent sur des interdictions d'épouser des parents caractérisés par leur degré de proximité généalogique.

Or, en reprenant la théorie de la parenté là où Lévi-Strauss l'avait laissée et en utilisant de façon pionnière l'outil informatique, Françoise Héritier a éclairé de façon décisive les principes de fonctionnement des structures d'alliance semi-complexes. Dans de tels systèmes, où des règles de mariage exclusivement négatives paraissent autoriser une grande liberté dans le choix du conjoint, elle a pu révéler l'existence d'unions préférentielles répétées à intervalles réguliers et de circuits stables d'échanges matrimoniaux entre groupes de diverses natures, phénomènes en apparence purement statistiques, mais qui obéissent en fait à des principes rigoureux quoique non explicitement formulés. On observe en effet que les mariages vont se nouer de façon préférentielle avec des parents situés juste après ceux qui sont interdits, aboutissant ainsi à ce que des alliés de consanguins renouvellent une union matrimoniale effectuée plusieurs générations auparavant entre des membres de leur parentèle sans pourtant avoir conscience qu'ils pratiquent ainsi un renchaînement d'alliance.

Les mécanismes de fonctionnement des structures semi-complexes d'alliance mis en évidence par Françoise Héritier sont eux-mêmes une expression de lois plus fondamentales, opérant également dans les structures élémentaires et qui, sur la base des invariants biologiques des humains, combinent critères de sexe et de consanguinité reconnue pour engendrer les diverses formules logiquement possibles de conceptualisation des liens de parenté et de mise en œuvre de l'alliance de mariage. Ces formules sont en grande partie l'effet des représentations que les humains se font des substances et des processus physiologiques, le deuxième axe de recherche auquel Françoise Héritier a apporté des contributions fondamentales. Dans toutes les sociétés, on s'accorde en effet pour reconnaître que la parenté est fondée sur l'échange ou le mélange de substances, que celles-ci soient corporelles (le sang, les os, la chair, le lait, les liquides sexuels), matérielles (la nourriture) ou immatérielles (le nom, l'âme des ancêtres), en sorte que l'apparentement se construit à partir de ces échanges directs ou indirects, réels ou symboliques, à l'étude desquels Françoise Héritier a consacré plusieurs années de son enseignement, malheureusement encore inédit.

Dans toute société, également, la différence entre le masculin et le féminin apparaît comme un gabarit conceptuel pour penser d'autres systèmes de différences, définir les frontières de ce qui est identique à soi ou non, de ce qui est compatible ou 
pas. D'où la prohibition de l'inceste qui interdit le rapprochement physique de personnes qui sont réputées partager des substances identiques, un redoublement porteur de danger pour la société tout entière, mais aussi, dans bien des parties du monde, pour l'équilibre cosmique. Ces considérations ont notamment amené Françoise Héritier à soutenir que l'inceste qu'elle a appelé du « second type » (entre des parents définis comme consanguins à travers des rapports sexuels avec une tierce personne) relevait d'un cumul de l'identique de même nature que l'inceste commun, du premier type. Allant plus loin que l'analyse combinatoire des substances, elle s'est enfin attachée sans relâche à établir les conséquences d'une asymétrie plus fondamentale, celle qu'elle a appelé la «valence différentielle des sexes » et qui pose partout le masculin comme supérieur au féminin. Cette inégalité, dont elle disait que son universalité ne la rend pas pour autant inéluctable, elle en a traqué les expressions multiples dans les domaines symboliques, politiques et religieux, fournissant une source d'inspiration et une référence pour toutes celles et ceux qui ont eu à cœur de lutter contre les discriminations sexistes. L'ensemble des recherches de Françoise Héritier, exposées dans une douzaine de livres et de très nombreuses publications scientifiques, lui a valu de multiples distinctions : la médaille d'argent du CNRS, le prix Irène Joliot-Curie, les grades de grand officier dans l'ordre de la Légion d'honneur, de grand-croix dans l'ordre du Mérite et de commandeur dans l'ordre des Arts et des Lettres, ainsi que des doctorats honoris causa de l'Université libre de Bruxelles et de l'université de Lausanne.

Enfin, Françoise Héritier était une chercheuse engagée qui, par ses écrits, ses conférences, ses interventions dans les médias, et dans les fonctions officielles qu'elle a occupées, s'est constamment efforcée de diffuser les progrès scientifiques de l'anthropologie de façon à ce qu'ils permettent une meilleure intelligence des enjeux contemporains et contribuent ainsi à dissiper les préjugés. En attestent, entre autres, le rôle qu'elle a joué comme présidente du Conseil national du sida, comme membre du Comité consultatif national d'éthique pour les sciences de la vie, comme membre du Haut-Conseil de la francophonie et du Haut-Conseil de la population et de la famille ou comme membre du Conseil économique et social. Gravement handicapée depuis plus de trente ans par une maladie auto-immune, cette femme exceptionnelle qui n'avait jamais cédé au découragement a offert un exemple peu commun d'alliance entre une acuité scientifique remarquable, une détermination aussi douce qu'inébranlable et une générosité rare vis-à-vis de tous ceux à qui elle a prodigué son enseignement, ses conseils et son amitié.

Pr Philippe DESCOLA, 25 mars 2018 